\title{
Qualité des œufs de consommation de trois types génétiques de poules commercialisés dans l'Est algérien
}

\author{
Moula, N.1,2@
}

\begin{abstract}
'Département des Productions Animales, Faculté de Médecine vétérinaire, Université de Liège, Liège, Belgique. 2Institut Vétérinaire Tropical, Faculté de Médecine vétérinaire, Université de Liège, Liège, Belgique.
\end{abstract}

\section{MOTS CLÉS SUPPLÉMENTAIRES}

Qualité de l'œuf.

Rapport jaune/Blanc.

Solidité de la coquille.

Races locales.

Algérie.

\section{RÉSUMÉ}

Le but de cette étude consiste à comparer la qualité, en termes de composition et de solidité, des œufs de poules commercialisés sur les marchés populaires de l'est algérien. Au total, 5004 œufs issus de trois types génétiques (poules locales -PL, poules industrielles -PI- et poules issues de croisement de poules locales et poules industrielles-PLPI-) ont été analysés. La souche industrielle a présenté des œufs dont le poids entier et le poids du blanc ainsi que la force maximale de rupture de la coquille étaient significativement $(\mathrm{P}<0,001)$ supérieurs à ceux des autres génotypes. Les œufs de poules locales, malgré un poids inférieur aux autres races $(\mathrm{PL}: 54,15 \mathrm{~g}, \mathrm{PLPI}: 59,74 \mathrm{~g}$ et PI : $66,62 \mathrm{~g} ; \mathrm{P}<0,05$ ), étaient supérieurs en terme de rapport jaune/blanc (PL : 0,57, PLPI : 0,50 et $\mathrm{Pl}: 0,44 ; \mathrm{P}<0,05)$, ce qui représente un critère de qualité majeur. La force maximale de rupture de la coquille était significativement $(P<0,05)$ inferieure chez les poules locales (PL : 35,99n, PLPI : $36,76 \mathrm{n}$ et $\mathrm{PI}: 37,30 \mathrm{n} ; \mathrm{P}<0,05)$. Des coefficients de corrélations significatifs $(\mathrm{P}<0,001)$ ont été enregistrés entre le Poids de l'œuf et les poids du blanc $(95,2 \%)$, du jaune $(50,2 \%)$ et de la coquille $(68,5 \%)$, les proportions du blanc $(79,2 \%)$, proportions du jaune $(-74,4 \%)$ et les proportions de la coquille $(-36,6 \%$ ) l'épaisseur moyenne de la coquille $(59,6 \%)$, les unités d'Haugh $(-17,55)$, I'indice de forme $(19,1 \%)$, le rapport jaune / blanc $(-75,3 \%)$ et de la force maximale de rupture de la coquille $(10,1 \%)$. En conclusion, l'origine des œufs commercialisés (PL, PI et PLPI) influe de façon significative la composition et la qualité des œufs commercialisés en Algérie.

\section{Quality Assessment of eggs of 3 chicken lines Marketed in Eastern Algeria}

\section{SUMMARY}

\section{ADDITIONAL KEYWORDS}

Egg quality.

Yolk/Albumen ratio.

Shell strength.

Local breed.

Algeria.

\section{INFORMATION}

\section{Cronología del artículo.}

Recibido/Received: 21.12.2016

Aceptado/Accepted: 05.05.2018

On-line: 15.07 .2018

Correspondencia a los autores/Contact e-mail:

Nassim.Moula@ulg.ac.be
The aim of this study was to compare the commercial eggs quality in eastern Algeria. A total of 5004 eggs from three genetic types (local hens -PL, industrial -PF- hens and chickens from crossing local chickens and industrial-PLPt-hens) were analyzed. Eggs weight, albumen weight and maximum breaking strength of the shell from the industrial strain were significantly higher than those from other genotypes $(P<0.001)$. Eggs from local chickens, despite a lower weight to other genotypes (PL: 54.15g; PLPI: $59.74 \mathrm{~g}$; Pl: 66.62g; P $<0.05)$ while yellow/albumen ratio $(0.57,0.50$ and 0.44 respectively) was higher $(P<0.05)$. Maximum breaking strength of the shell was significantly $(\mathrm{P}<0.05)$ lowest in $\mathrm{PL}(35,99 \mathrm{~N})$ compared to PLPI $(36.76 \mathrm{~N})$ and PI (37.30N). Significant correlation coefficients $(P<0.001)$ were recorded between egg weight and albumen weight $(0.95)$, yolk weight $(0.50)$, shell weight $(0.69)$, albumen percentage $(0.79)$, proportions of yellow $(-0.74)$ and shell percentage $(-0.37)$, the average thickness of the shell $(0.60)$, Haugh units $(-0.18)$, shape index (0.19), yellow / albumen ratio $(-0.75)$ and the maximum breaking strength of the shell $(0.10)$. The origin of the eggs (PL, PLPI and PI) influences significantly on composition and quality of commercial eggs in eastern Algeria.

\section{INTRODUCTION}

Depuis des milliers d'années, les œufs d'oiseaux et en particulier l'œuf de poule constituent pour l'homme un aliment essentiel (Nau et al., 2003). L'œuf est un aliment naturel parfait, appartenant à cette rare catégorie des aliments protéiques complets. Il contient les neuf acides aminés essentiels que l'organisme est incapable de synthétiser (Stadelman et Pratt, 1989). Il constitue une ressource protéique, lipidique, minérale et vitaminique facilement renouvelable. Les protéines sont indispensables à la formation et à l'entretien des tissus de l'organisme humain ainsi qu'à la bonne croissance et au bon développement de celui-ci. De 
plus, ils sont à l'origine de la formation des muscles, des organes, de la peau, des cheveux, tout comme celle des anticorps, des enzymes et des hormones : tous sont formés de protéines (Agriculture et Agroalimentaire Canada, 2006).

Le faible prix de revient des œufs en fait une source de protéines et de lipides animales de faible coût (Nys et Sauveur, 2004). De plus ils sont acceptés dans le monde entier et ne font l'objet d'aucune interdiction culturelle ou religieuse (Bessadok et al., 2003).

L'évolution du mode de vie et des habitudes de consommation, accompagnée du développement de la restauration rapide, $n^{\prime}$ a fait qu'augmenter sa demande. En effet les protéines de l'œuf sont des ingrédients appréciés dans beaucoup d'aliments (Mine, 2002).

De nos jours, les œufs sont plus qu'une source d'alimentation diététique. Leurs effets antioxydant, cryoprotecteur, antibactérien, antiviral, antihypertenseur, émulsifiant et coagulant (Mine, 2002 ; Mine et Kovacs-Nolan, 2004 ; Nau et al., 2003) ont permis leur utilisation dans des secteurs industriels très diversifiés (agroalimentaire, pharmaceutique, cosmétique, etc.).

La production et la consommation mondiale d'œufs ont triplé depuis les années soixante et continuent d'augmenter régulièrement. Face à cette demande croissante, la différenciation de la composition des œufs peuvent jouer un rôle important dans la diversification de son marché, aussi bien pour les œufs frais que pour les œufs traités industriellement.

L'objectif de cette étude est d'étudier les caractéristiques des œufs mis en vente sur les marchés publics dans l'est algérien.

\section{MATERIEL ET MÉTHODES}

Des œufs de commerce au nombre de 5004, de poules de trois types génétiques (poules locales (1), croisement poules locales $x$ poules industrielles (2) et poules industrielles (3) ont été étudiés. Les œufs provenaient de marchés populaires de 5 wilayets (Bejaia, Jijel, Mila, Sétif et Constantine) de l'Est algérien. L'étude s'est déroulée entre les mois de novembre 2014 et Février 2015. Les analyses ont été effectuées au lendemain de l'achat des œufs.

\section{Mesure de LA QuAltí des oevfs}

La qualité externe et interne des œufs a été contrôlée. Après la numérotation des œufs, des mesures allant de l'indice de forme au poids des différents composants de l'œuf, à la force maximale de rupture de la coquille et aux unités Haugh ont été réalisées.

\section{INDICE DE FORME}

La longueur et la largeur de l'œuf sont mesurées par un pied à coulisse électronique, il s'agit de déterminer l'indice de forme de l'œuf, défini par Parmar et al., (2006) et Monira et al., (2003) comme étant le rapport de la largeur sur la longueur de l'œuf multiplié par 100.

\section{ForCE MAXIMALE DE RUPTURE DE LA COQUILLE (FMAX(N))}

La résistance de la coquille (en Newton) a été estimée par la méthode de la force nécessaire à la rupture pendant la compression quasi-statique. Elle a été mesurée en utilisant une machine universelle de traction/compression décrite par Moula et al., (2010). Les œufs ont été placés horizontalement entre deux plaques plates parallèles en acier et comprimés à la vitesse de $10 \mathrm{~mm} / \mathrm{min}$. La mesure consiste à appliquer une force progressive sur l'œuf et ne s'arrête qu'au moment de la rupture de la coquille correspondant à la force maximale.

\section{UNITÉS D'HAUGH}

Les Unités d'Haugh permettent d'estimer la fraîcheur de l'œuf. L'appareil utilisé pour cette mesure est composé d'une balance électronique, d'un micromètre tripode (tous les deux reliés à un ordinateur) et $d$ 'une surface en verre présentant une légère pente.

Chaque œuf a été pesé et cassé sur une surface en verre. L'épaisseur de l'albumen a été mesurée avec le micromètre tripode, au niveau de la hauteur maximale.

L'ordinateur enregistre les résultats et calcule les unités d'Haugh en appliquant la formule décrite par Haugh (1937):

$$
\begin{aligned}
& \mathrm{HU}=100 \log \left(\mathrm{H}-1,7 \mathrm{~W}^{0,37}+7,6\right) \\
& \mathrm{HU}=\text { unités d'Haugh. } \\
& \mathrm{H}=\text { hauteur de l'albumen en } \mathrm{mm} . \\
& \mathrm{W}=\text { poids de l'œuf en } \mathrm{g} . \\
& \text { POIDS DES DIFFÉRENTS COMPOSANTS DE L'OEUF }
\end{aligned}
$$

\section{POIDS DES DIFFÉRENTS COMPOSANTS DE L'OEUF}

Conjointement à la mesure des unités d'Haugh, le poids de la coquille (avec les membranes coquillières) a été déterminé, puis le jaune été séparé du blanc sur une surface en verre avant d'être pesé.

Le poids du blanc est obtenu par soustraction des poids du jaune et de la coquille du poids de l'œuf entier.

\section{EPAISSEUR DE LA COQUILLE}

L'épaisseur de la coquille a été mesurée, après le prélèvement des membranes coquillières sur trois points de l'équateur, avec un micromètre électronique. La moyenne de ces trois valeurs est considérée dans l'analyse des données. Tyler et Geake (1964) ont rapporté que la coquille de l'œuf est plus fine mais presque uniforme en zone équatoriale.

\section{AUTRES PARAMĖTRES}

D'autres paramètres ont été également déterminés :

- du rapport jaune $/$ blanc $=$ poids du jaune $/$ poids d'albumen

- de la proportion du blanc $=$ (poids du blanc $/$ poids de l'œuf entier)* ${ }^{*} 100$

- de la proportion du jaune $=$ (poids du jaune / poids de l'œuf entier)*100

- de la proportion de la coquille $=$ (poids de la coquille / poids de l'œuf entier ${ }^{*} 100$ 


\section{ANALYSE STATISTIQUE}

L'analyse statistique a été réalisée avec le logiciel SAS (Statistical Analysis System, 1989). Le modèle linéaire généralisé (GLM) a été utilisé pour effectuer une analyse de variance sur chaque paramètre, afin de déterminer les différences qui existent entre les trois types génétiques étudiés et leurs significations statistiques. Pour chaque paramètre, une moyenne « moindres carrés » ainsi que l'erreur standard ont été calculées.

Les corrélations existant entre les différents caractères ont été également étudiées.

\section{RÉSULTATS}

\section{LA FORME ET LA SOLIDITÉ DE LA COQUILLE}

Le Tableau I indique les pourcentages des œufs fêlés et ou fissurés au niveau de la coquille. Aucune différence significative $(P>0,05)$ n'a été observée entre les trois types génétiques.

Un résumé des mesures effectuées sur la solidité de l'œuf est donné dans le Tableau II.

Des différences hautement significatives $(\mathrm{P}<0,001)$ ont été enregistrées dans l'épaisseur des coquilles d'œufs de PI $(0,371 \mathrm{~mm})$, PLPI $(0,320 \mathrm{~mm})$ et PL $(0,312 \mathrm{~mm})$. Pour ce qui concerne la conformation de l'œuf, des différences hautement significatives $(\mathrm{P}<0,001)$ ont été enregistrées entre les différents génotypes. Les souches Industrielles sont caractérisées par les œufs les plus longs $(59,22 \mathrm{~mm})$ et les plus larges (45,01 mm) ; alors que les poules locales avaient montré les œufs les plus petits avec respectivement 55,80 mm de longueur et 41,87 $\mathrm{mm}$ de largeur. Les œufs issus du croisement Industrielles x Locales avait présenté des valeurs intermédiaires (Longueur : 56,14 mm, Largeur : $43,71 \mathrm{~mm})$.

Les différences observées pour l'indice de forme des œufs des différentes poules ont été très significatives $(\mathrm{P}<0,001)$ (Tableau II).

Des différences hautement significatives $(P<0,001)$ ont été observées pour la force maximale de rupture de la coquille des œufs des différents groupes génétiques. Les poules industrielles avaient présenté les œufs les plus solides $(37,30 \mathrm{~N})$, alors que les poules locales avaient présenté les œufs les moins solides avec une résistance à une force de rupture de 35,99 N. Le croisement Industrielles $x$ Locales avait présenté une valeur moyenne intermédiaire $(36,76 \mathrm{~N})$.
LE POIDS ET LES PROPORTIONS DES DIFFÉRENTS COMPOSANTS DE L'OEUF

Le poids de l'œuf et les proportions du blanc, du jaune et de la coquille sont présentés dans le Tableau III. Des différences hautement significatives $(\mathrm{P}<0,001)$ ont été observées, entre les génotypes, pour les poids de l'œuf entier, du blanc, du jaune et de la coquille. Les poules industrielles ont donné des œufs avec un poids entier significativement supérieurs aux autres génotypes $(66,62 \mathrm{~g}$ contre $59,74 \mathrm{~g}$ et $54,15 \mathrm{~g}$ pour respectivement les PLPI et PL). Des observations similaires ont été enregistrées pour les poids de l'albumen, du jaune et de la coquille.

Les différences enregistrées dans les proportions des différents composants de l'œuf sont hautement significatives $(P<0,001)$ entre les trois génotypes. Les poules locales qui présentaient le plus faible taux d'albumen $(55,97 \%)$ avaient montré le pourcentage le plus élevé de jaune $(31,65 \%)$ et de coquille (12,38 \%). Parallèlement, les souches commerciales qui présentaient également le pourcentage le plus important de blanc $(61,75 \%)$ avaient donné le plus faible taux de jaune $(26,92 \%)$ et de coquille (11,33\%). Le croisement poules Industrielles x Locales avait enregistré $29,20 \%$ de jaune, 58,77 \% de blanc et $12,03 \%$ de coquille.

\section{LE RAPPORT JAUNE BLANC ET LA FRAîCHEUR DE L'OEUF}

La poule locale a présenté le rapport jaune/blanc le plus important $(0,57$ contre 0,50 pour les poules croisées et 0,44 pour les poules industrielles) (Tableau III).

Comme indiqué dans le Tableau II, des œufs avec un albumen étalé ont été observés. Cependant aucune différence significative $(P>0,05)$ n'a été observée entre leurs pourcentages chez les trois génotypes.

Pour la hauteur d'albumen, celle-ci varie entre 6,76 $\mathrm{mm}$ pour les poules industrielles à $8,00 \mathrm{~mm}$ pour le croisement poules Industrielles x Locales. Les poules locales ont présenté une valeur intermédiaire $(7,30 \mathrm{~mm})$.

Pour les Unités d'Haugh, des différences hautement significatives $(\mathrm{P}<0,001)$ ont été observées entre les génotypes. Le croisement Industrielles x Locales a donné les œufs les plus frais avec 89,31 Unités d'Haugh contre 86,46 pour les poules locales et 79,65 pour les poules industrielles.

\section{Les Coefficients de CORrÉlation de PeARSON entre les DIFFÉRENTES VARIABLES ÉTUDIÉES}

Les coefficients de corrélation de Pearson sont présentés dans le Tableau IV. Le poids et les proportions

Tableau I. Nombre et pourcentage d'œufs fêlés et ou cassés par race (Number and percentage of eggs cracked and/or broken by breed).

\begin{tabular}{cccc}
\hline & Race: nombre (\%) & Valeur de Chi $^{2}$ & Signification \\
\hline Industrielle & Industrielle $x$ Locale & Locale & \\
\hline
\end{tabular}

CEufs fêlés et ou cassés

$92(5,96)$

$108(6,59)$

$120(6,62)$
0,54

CEufs avec un albumen étalé
$50(3,03)$

$52(2,87)$
0,75 
Tableau II. Moyennes des moindres carrés et erreurs standards (LSM \pm SE) de la moyenne de l'épaisseur de la coquille, de la longueur et de la largeur de l'œuf, de l'indice de forme et de la force maximale de rupture de la coquille (Averages of least squares and standard errors $(\mathrm{LSM} \pm \mathrm{SE}$ ) of the mean of the shell thickness, the length and width of the egg, the shape index and the maximum strength of the shell rupture).

\begin{tabular}{|c|c|c|c|c|}
\hline & Industrielle & Industrielle $\mathrm{x}$ Locale & Locale & $R^{2}(\%)$ et signification \\
\hline$n$ & 1544 & 1648 & 1812 & \\
\hline Epaisseur de la coquille $\left(10^{-2} \mathrm{~mm}\right)$ & $37,09 \pm 0,08^{a}$ & $32,04 \pm 0,08^{b}$ & $31,21 \pm 0,07^{b}$ & $41,10^{* * *}$ \\
\hline Longueur de l'œuf (mm) & $59,22 \pm 0,06^{a}$ & $56,14 \pm 0,05^{b}$ & $55,80 \pm 0,04^{c}$ & $33,07^{* \star *}$ \\
\hline Largeur de l'œuf (mm) & $45,01 \pm 0,04^{a}$ & $43,71 \pm 0,04^{b}$ & $41,87 \pm 0,05^{c}$ & $38,93^{* * *}$ \\
\hline Indice de forme & $76,09 \pm 0,08^{a}$ & $77,91 \pm 0,06^{b}$ & $75,10 \pm 0,07^{c}$ & $13,24^{\star \star *}$ \\
\hline $\mathrm{n}$ & 1454 & 1538 & 1692 & \\
\hline Force maximale de rupture (newton) & $37,30 \pm 0,16^{a}$ & $36,76 \pm 0,14^{b}$ & $35,99 \pm 0,15^{c}$ & $74,0^{* * *}$ \\
\hline
\end{tabular}

Sur la même ligne, les valeurs avec des lettres différentes présentent une différence significative au seuil de $P<0,0001 ;{ }^{* * *}: P<0,0001$.

d'albumen, du jaune et de la coquille sont en forte corrélation avec le poids de l'œuf.

Les corrélations du poids de l'œuf avec l'épaisseur de la coquille $(0,60)$, la longueur $(0,77)$ et la largeur $(0,82)$ de l'œuf sont importantes. Par contre, celle avec l'indice de forme n'est que de 0,19.

Une forte corrélation négative $(-0,75)$ a été enregistrée entre le rapport jaune / blanc et le poids de l'œuf entier traduisant une diminution du rapport quand le poids de l'œuf augmente.

Le poids du blanc est très fortement corrélé avec la majorité des paramètres étudiés $(0,39$ avec le poids du jaune, 0,57 avec le poids de la coquille, 0,89 avec le pourcentage du blanc, $-0,73$ avec le pourcentage du jaune, 0,79 avec la longueur de l'œuf, 0,89 avec la largeur de $l^{\prime} œ u f,-0,84$ avec le rapport jaune / blanc et 0,11 avec la force maximale de rupture de la coquille).

Les corrélations du poids du jaune sont significatives $(\mathrm{P}<0,001)$ avec le poids $(0,53)$ et le pourcentage $(-0,27)$ de la coquille, la longueur $(0,41)$ et la largeur $(0,46)$ de l'œuf et le rapport jaune / blanc $(0,16)$. Le poids de la coquille est fortement $(\mathrm{P}<0,001)$ corrélé avec les pourcentages du blanc $(0,37)$, du jaune $(-0,44)$, l'épaisseur moyenne de la coquille $(0,48)$, la longueur $(0,55)$ et la largeur $(0,47)$ de l'œuf, le rapport jaune / blanc $(-0,49)$ et la force maximale de rupture de la coquille $(0,15)$.

Les pourcentages du blanc sont fortement $(\mathrm{P}<0,001)$ corrélés avec les pourcentages du jaune $(0,92)$, de la coquille $(-0,55)$, l'épaisseur moyenne de la coquille $(0,45)$ et le rapport jaune / blanc $(-0,97)$.

Des corrélations importantes $(\mathrm{P}<0,001)$ sont enregistrées entre le pourcentage du jaune et le pourcentage de la coquille $(0,29)$, l'épaisseur moyenne de la coquille $(-0,56)$, la longueur $(-0,65)$ et la largeur $(-0,56)$ de l'œuf et le rapport jaune / blanc $(0,99)$.

L'épaisseur moyenne de la coquille a présenté de fortes corrélations $(\mathrm{P}<0,001)$ avec le rapport jaune / blanc $(-0,54)$ et la force maximale de rupture $(0,28)$.

Enfin une corrélation $(-0,16)$ hautement significative $(\mathrm{P}<0,001)$ est enregistrée entre la force maximale de rupture et le rapport jaune / blanc.

\begin{tabular}{|c|c|c|c|c|}
\hline Paramètres & Industrielle & Industrielle $x$ Locale & Locale & $\mathrm{R}^{2}(\%)$ et signification \\
\hline $\mathrm{n}$ & 1544 & 1648 & 1812 & \\
\hline Poids de l'œuf (g) & $66,62 \pm 0,14^{a}$ & $59,74 \pm 0,14^{b}$ & $54,15 \pm 0,13^{c}$ & $46,15^{\star * *}$ \\
\hline Poids d'albumen (g) & $41,20 \pm 0,11^{a}$ & $35,20 \pm 0,11^{b}$ & $30,42 \pm 0,10^{c}$ & $50,30^{* * *}$ \\
\hline Poids du jaune $(\mathrm{g}$ ) & $17,88 \pm 0,04^{a}$ & $17,37 \pm 0,04^{b}$ & $17,05 \pm 0,04^{c}$ & $4,76^{\star * *}$ \\
\hline Poids de la coquille (g) & $7,54 \pm 0,02^{\mathrm{a}}$ & $7,17 \pm 0,02^{\mathrm{b}}$ & $6,68 \pm 0,02^{c}$ & $17,27^{* \star *}$ \\
\hline$\%$ albumen & $61,75 \pm 0,07^{a}$ & $58,77 \pm 0,07^{b}$ & $55,97 \pm 0,07^{c}$ & $39,74^{* * *}$ \\
\hline$\%$ jaune & $26,92 \pm 0,07^{a}$ & $29,20 \pm 0,06^{b}$ & $31,65 \pm 0,06^{c}$ & $36,03^{* * *}$ \\
\hline$\%$ coquille & $11,33 \pm 0,03^{a}$ & $12,03 \pm 0,03^{b}$ & $12,38 \pm 0,02^{c}$ & $15,01^{* * *}$ \\
\hline Rapport jaune/ blanc (\%) & $43,77 \pm 0,18^{a}$ & $50,01 \pm 0,18^{b}$ & $57,07 \pm 0,17^{c}$ & $36,24^{* * *}$ \\
\hline $\mathrm{n}$ & 1504 & 1598 & 1760 & \\
\hline Hauteur d'albumen (mm) & $6,76 \pm 0,04^{a}$ & $8,00 \pm 0,04^{b}$ & $7,30 \pm 0,03^{c}$ & $12,09^{* * *}$ \\
\hline Unités d'Haugh & $79,65 \pm 0,23^{a}$ & $89,31 \pm 0,23^{b}$ & $86,46 \pm 0,22^{c}$ & $17,80^{* * *}$ \\
\hline
\end{tabular}

Sur la même ligne, les valeurs avec des lettres différentes présentent une différence significative au seuil de $P<0,0001 .{ }^{* * *}: P<0,0001$. 


\begin{tabular}{|c|c|c|c|c|c|c|c|c|c|c|c|c|c|}
\hline & 1 & 2 & 3 & 4 & 5 & 6 & 7 & 8 & 9 & 10 & 11 & 12 & 13 \\
\hline 1 & 1 & & & & & & & & & & & & \\
\hline 2 & $\underset{* * *}{95,2}$ & 1 & & & & & & & & & & & \\
\hline 3 & $\begin{array}{l}50,2 \\
* \star \star\end{array}$ & $\begin{array}{l}39,8 \\
* * *\end{array}$ & 1 & & & & & & & & & & \\
\hline 4 & $\begin{array}{l}68,5 \\
* * \star\end{array}$ & ${ }_{* * \star}^{57,2}$ & $\begin{array}{l}53,1 \\
* * \star\end{array}$ & I & & & & & & & & & \\
\hline 5 & $\begin{array}{l}79,2 \\
* * \star\end{array}$ & $\begin{array}{l}89,3 \\
* * \star\end{array}$ & $\begin{array}{l}-10,5 \\
\star * \star\end{array}$ & $\begin{array}{l}37,2 \\
* \star *\end{array}$ & I & & & & & & & & \\
\hline 6 & $\begin{array}{l}-74,4 \\
\star \star \star \star\end{array}$ & $\begin{array}{l}-73,1 \\
* * *\end{array}$ & 23,1 & -44.1 & $\begin{array}{l}-91,8 \\
* \star *\end{array}$ & I & & & & & & & \\
\hline 7 & $\begin{array}{l}-36,6 \\
* \star \star\end{array}$ & $\begin{array}{l}-56,1 \\
* * \star\end{array}$ & $\begin{array}{l}-27,2 \\
\star \star \star\end{array}$ & $\begin{array}{l}36,4 \\
* \star *\end{array}$ & $\begin{array}{l}-54,9 \\
* * \star\end{array}$ & $\underset{* * *}{28,7}$ & 1 & & & & & & \\
\hline 8 & $-17,5$ & $\begin{array}{l}-15,7 \\
* * \star\end{array}$ & $\begin{array}{l}-11,5 \\
\star \star \star\end{array}$ & $\begin{array}{l}-13,1 \\
* * *\end{array}$ & $-09,1$ & $\begin{array}{l}7,7 \\
* * *\end{array}$ & $\begin{array}{l}5,5 \\
* *\end{array}$ & I & & & & & \\
\hline 9 & $\begin{array}{l}59,6 \\
* \star \star\end{array}$ & $\begin{array}{l}58,2 \\
* * *\end{array}$ & $\begin{array}{l}8,05 \\
\star * \star\end{array}$ & $\begin{array}{l}48,1 \\
* \star *\end{array}$ & $\begin{array}{l}44,8 \\
\star \star \star\end{array}$ & $\begin{array}{l}-55,9 \\
* * *\end{array}$ & $\begin{array}{l}-17,2 \\
* * *\end{array}$ & $\begin{array}{l}-21,0 \\
* \star *\end{array}$ & 1 & & & & \\
\hline 10 & $\begin{array}{l}77,1 \\
* \star \star\end{array}$ & $\begin{array}{l}78,8 \\
* * \star\end{array}$ & $\begin{array}{l}40,7 \\
\star \star \star\end{array}$ & $\begin{array}{l}54,9 \\
* \star *\end{array}$ & $\begin{array}{l}59,3 \\
* * *\end{array}$ & $-65,1$ & $\begin{array}{l}-33,8 \\
* * *\end{array}$ & $\begin{array}{l}-7,7 \\
\star \star \star\end{array}$ & $\begin{array}{l}43,6 \\
* * \star\end{array}$ & I & & & \\
\hline 11 & $\underset{* * \star}{82,2}$ & $\underset{* * *}{89,3}$ & $\underset{* \star *}{45,9}$ & $\underset{* * \star}{47,1}$ & $\begin{array}{l}68,8 \\
* * *\end{array}$ & $\begin{array}{l}-56,4 \\
* * *\end{array}$ & $\begin{array}{l}-44,6 \\
* * *\end{array}$ & $\begin{array}{l}5,9 \\
* * *\end{array}$ & $\underset{* * \star}{49,2}$ & $\begin{array}{l}63,1 \\
* * *\end{array}$ & I & & \\
\hline 12 & $\underset{* * \star}{19,1}$ & $\underset{* \star \star}{20,4}$ & $\begin{array}{l}4,9 \\
* *\end{array}$ & $\underset{* \star *}{15,9}$ & $\begin{array}{l}19,8 \\
\star \star \star\end{array}$ & $-27,1$ & $\underset{* * *}{8,7}$ & $\underset{* * \star}{14,9}$ & $\begin{array}{l}8,9 \\
* * *\end{array}$ & $\begin{array}{l}-46,5 \\
* \star \star\end{array}$ & ${ }_{* * *}^{47,0}$ & 1 & \\
\hline 13 & $\begin{array}{l}-75,3 \\
* * \star\end{array}$ & $\begin{array}{l}-83.6 \\
* \star \star\end{array}$ & $\begin{array}{l}16,1 \\
* \star \star\end{array}$ & $\begin{array}{l}-49,2 \\
* \star *\end{array}$ & $\begin{array}{l}-96,9 \\
\star \star \star \\
* \star\end{array}$ & $\begin{array}{l}99,1 \\
* * \star\end{array}$ & $\underset{* * \star}{44,9}$ & $\begin{array}{l}-8,7 \\
* * *\end{array}$ & $\begin{array}{l}-53,9 \\
* \star \star\end{array}$ & $\begin{array}{l}-54,8 \\
* \star \star\end{array}$ & $\begin{array}{l}-68,9 \\
\star * *\end{array}$ & $\begin{array}{l}-21,0 \\
* * *\end{array}$ & I \\
\hline 14 & $\underset{* * \star}{10,1}$ & $\underset{* \star \star}{10,8}$ & $\begin{array}{l}0.7 \\
\text { ns }\end{array}$ & $\underset{* \star \star}{14,6}$ & $\begin{array}{l}9,24 \\
\star \star \star\end{array}$ & $\begin{array}{l}-12,6 \\
* \star *\end{array}$ & $\begin{array}{l}4,9 \\
* *\end{array}$ & $\begin{array}{l}7,8 \\
* * *\end{array}$ & $\underset{* \star \star}{28,0}$ & $\begin{array}{l}4,2 \\
* *\end{array}$ & $19,1^{\star \star \star}$ & $\underset{* \star \star}{15,6}$ & $\begin{array}{l}-12,7 \\
* * *\end{array}$ \\
\hline
\end{tabular}

***: $P<0,0001 ;{ }^{* *}: P<0,001 ; n s: P \geq 0,05$.

1 : poids de l'œuf entier ; 2 : poids d'albumen ; 3 : poids du jaune ; 4 : poids de la coquille ; $5: \%$ du blanc ; $6: \%$ du jaune ; $7: \%$ de la coquille ; 8 : unités d'Haugh; 9 : épaisseur moyenne de la coquille ; 10 : longueur de l'œuf ; 11 : largeur de l'œuf ; 12: indice de forme ; 13 : rapport jaune/ blanc ; 14 : force maximale de rupture de la coquille.

\section{DISCUSSION}

\section{LA FORME ET LA SOLIDITÉ DE L'OEUF}

Les proportions des œufs défectueux (fêlés et / ou fissurés) des trois génotypes sont similaires. Si cette situation peut être expliquée probablement en partie par le type d'élevage qui est semblable chez les poules locales et les poules croisées (élevage au sol), aucune explication ne peut être apportée pour les poules industrielles élevées en batterie.

Pour l'épaisseur de la coquille, la différence est très significative $(P<0,001)$ entre les trois génotypes. Les poules locales ont montré des valeurs plus faibles par rapport aux poules industrielles et aux poules croisées. Suk et Park (2001) ont observé à travers leur étude sur les races KNC (Korean Native Chicken) et CEC (Commercial egg-type chicken) qu'à l'âge de 55 semaines, les œufs de la souche commerciale présentaient des coquilles plus épaisses que celles de la race locale $(0,42$ $\mathrm{mm}$ contre $0,34 \mathrm{~mm}$ ). Dans une autre étude, Offiong et al., (2006), ont rapporté des valeurs de $0,34 \mathrm{~mm}$ et $0,36 \mathrm{~mm}$ pour les poules de souches commerciales et les poules de races locales. Benabdeljelil et al. (2003) marqué dans leur étude sur les croisements race locale et souche commerciale (Fayoumi $\times$ Leghorn, ISA $\times$
Mandarah) des coquilles moins épaisses avec respectivement $0,388 \mathrm{~mm}$ et $0,394 \mathrm{~mm}$ contre $0,394 \mathrm{~mm}$ pour la souche commerciale. Kemps et al., (2006) ont rapporté que des œufs à poids entier supérieur $(65,2 \mathrm{~g}$ pour les poules de race Bovans) présentaient des coquilles plus épaisses $(0,353 \mathrm{~mm})$ que celles de la race Hissex $(0,351$ $\mathrm{mm}$ ) qui pesaient $59,9 \mathrm{~g}$.

Pour la longueur et la largeur de l'œuf, mesurées dans le but de calculer l'indice de forme, une différence hautement significative $(\mathrm{P}<0,001)$ est enregistrée entre les races locales et les souches commerciales. Premavalli et Viswanathan (2004) et Ôzbey et Esen (2007) expliquent ces variations par le mode d'élevage avec le système en cage qui favorise la production d'œufs avec des indices de forme élevés. Cela peut expliquer en partie la supériorité de l'indice de forme de la souche industrielle par rapport aux poules locales. Cependant, ces résultats ne correspondent pas à ceux de Amer (1972) et Merat et al., (1983) qui ont noté dans leurs travaux que les races locales Fayoumi et Dandarawi ont un indice supérieur à celui de la souche commerciale Rhode Island Red. Ces variations sont plus de nature génétique. D'ailleurs Dottavio et al. (2001) ont enregistré d'importantes différences dans l'indice de 
forme des œufs des différents réservoirs génétiques qu'ils ont étudiés.

En ce qui concerne la force maximale de rupture de la coquille (Fmax) les différences observées entre les génotypes étudiés sont hautement significatives $(\mathrm{P}<0,001)$. Les poules industrielles avaient présenté une Fmax supérieure aux autres génotypes. De même, Tixier-Boichard et al., (2006a) ont noté des valeurs similaires à la présente étude, $35,00 \mathrm{~N}$ pour la race locale Fayoumi et $36,70 \mathrm{~N}$ pour la souche commerciale Isa Brown. Cette différence peut être expliquée par le fait que les souches commerciales ont fait l'objet d'une sélection poussée pour améliorer la solidité de la coquille contrairement aux poules locales algériennes qui n'ont subi aucune sélection.

\section{LE POIDS ET LES PROPORTIONS DES DIFFÉRENTS COMPOSANTS DE L'OEUF}

\section{LE POIDS DE L'OEUF}

Des différences hautement significatives $(P<0,001)$ sont enregistrées dans le poids des œufs des trois génotypes étudiés. Les poules industrielles ont produit les œufs les plus lourds avec un poids de 66,62 $\mathrm{g}$ contre $59,74 \mathrm{~g}$ pour les poules locales et $54,15 \mathrm{~g}$ pour les poules croisées. Ces résultats sont en parfaite concordance avec ceux de Amer (1972) qui a observé un poids d'œufs inférieur pour les races locales Fayoumi $(42,15 \mathrm{~g})$ et Dandarawi $(42,70 \mathrm{~g})$ comparées à la souche commerciale Rhode Island Red (51,89 g). De même Tixier-Boichard et al., (2006b) ont enregistré des résultats similaires à la présente étude, avec la race locale Fayoumi (42,8 g) et la souche Isabrown ( $58,8 \mathrm{~g})$. De plus Parmar et al., (2006) ont enregistré dans leur étude sur les œufs de race de poule indigène Kadaknath que le poids des œufs était inférieur à 45,41 g. De même, les onze souches commerciales étudiées par Harms et Hussein (1993) avaient un poids moyen de plus de 59 g. En effet, le type commercial de poules pondeuses a bénéficié d'une sélection intensive depuis des générations, qui doit à priori lui donner un avantage pour les principaux caractères tels que le poids de l'œuf, du blanc, de la coquille et du jaune (Hocking et al., 2003 ; Benabdeljelil et Merat, 1995).

\section{LE POIDS DU BLANC}

Dans la présente étude, les résultats obtenus pour le poids du blanc sont confortés par les résultats de Offiong et al., (2006), Tixier-Boichard et al., (2006b) et Benabdeljelil et al., (2003) qui ont rapporté des valeurs élevées du poids du blanc chez les souches commerciales comparées aux races locales. Cette supériorité peut être expliquée par le poids de l'œuf entier, important chez les lignées commerciales ayant subi une importante sélection génétique. De plus, une corrélation positive très significative est enregistrée entre le poids de l'œuf entier et du blanc. Hartmann et al., (2000) ont rapporté que le poids du blanc augmente avec l'augmentation de celui de l'œuf entier.

\section{LE POIDS DU JAUNE}

Les différences observées sur le poids du jaune avec la souche industrielle, qui a présenté un poids plus élevé, s'explique par celui de l'œuf entier des souches commerciales. De même Benabdeljelil et Merat (1995) avaient observé une différence significative $(\mathrm{P}<0,001)$ entre la Fayoumi $(16,18 \mathrm{~g})$ et la Rhode Island Red $(18,88 \mathrm{~g})$. D'après les résultats de Harms et Hussein (1993) et de Parmar et al. (2006), les œufs de poules locales présentaient un poids de jaune inférieur à $15,38 \mathrm{~g}$ alors que les œufs de poules commerciales dépassaient largement cette valeur (à l'exception de l'IsaBrown et de Hy-Line W36). Comme le poids du blanc, le poids du jaune est positivement et fortement corrélé avec le poids de l'œuf entier.

\section{LE POIDS DE LA COQUILLE}

Une différence significative $(\mathrm{P}<0,001)$ est enregistrée entre le poids des coquilles d'œufs issus des trois types de poules étudiés. Les poules commerciales ont présenté les coquilles les plus lourdes. Cela pourrait s'expliquer par le poids de l'œuf entier des souches commerciales ainsi que par la sélection génétique. Suk et Park (2001) ont noté que les coquilles de poule KNC (Korean Native Chicken) ont présenté un poids inférieur à celui de la poule CEC (Commercial egg-type chicken).

D'autres études ont lié le poids de la coquille à la race. Dans ce contexte, Amer (1972) avait enregistré un poids de coquille pour la Fayoumi supérieur à celui de la Rhode Island Red (4,4 g contre 4,3 g). Dans les études de Fletcher et al., (1983) et de Harms et Hussein (1993), le poids de la coquille variait d'une souche à une autre sans lien avec le poids de l'œuf entier.

\section{LE POURCENTAGE DU BLANC}

Le pourcentage du blanc est significativement $(P$ $<0,001)$ différent entre les trois génotypes étudiés. La souche industrielle a présenté le pourcentage le plus élevé $(61,75 \%)$ alors que les poules locales ont affiché de loin le pourcentage le plus faible (55,97\%). Merat et al., (1983) et Amer (1972) ont constaté que la Fayoumi avait enregistré un pourcentage d'albumen inférieur à celui de la Rhode Island Red. De même, Tixier-Boichard et al., (2006b) ont observé un pourcentage de 63 $\%, 55,3 \%$ et $58,3 \%$ pour respectivement $l^{\prime}$ Isa Brown, la Fayoumi et le croisement des deux races (Isa Brown $x$ Fayoumi).

Tharrington et al., (1999) ont rapporté que l'augmentation du poids de l'œuf entier lors de la sélection de lignées commerciales s'accompagne d'une élévation des proportions du blanc au détriment de celles du jaune qui diminuent malgré l'augmentation de son poids. De plus, Jaya Laxmi (2006) a noté une corrélation génétique positive $(0,256)$ entre le poids de l'œuf entier et le pourcentage du blanc.

\section{LE POURCENTAGE DU JAUNE}

Parce qu'elle est liée à un plus fort taux de matière sèche dans l'œuf et aussi à un apport plus important d'acides gras essentiels, une proportion de jaune plus élevée peut-être considérée comme favorable du point de vue de la valeur nutritive de l'œuf (Moula et al., 2010 ; 2013). En effet, la proportion élevée du jaune est l'une des plus importantes qualités internes de l'œuf. Dans cette étude, les poules locales avaient présenté le plus important pourcentage (31,65\%) suivi des poules 
croisées (29,20\%). Les poules industrielles ont présenté un pourcentage de $26,92 \%$ du jaune. Ces différences hautement significatives $(\mathrm{P}<0,001)$ entre les poules locales et les poules industrielles confirment les résultats obtenus par Tixier-Boichard et al., (2006b) et Amer (1972) qui ont enregistré chez la race locale Fayoumi des pourcentages de jaune variant de 30,87 à 34,80\% contre des valeurs allant de 26,60 à 29,69 \% pour les souches commerciales. Dans l'étude de Padhi et al., (1998), les proportions du jaune variaient de 30,77\% à $33,18 \%$ pour les races locales contre $26,86 \%$ pour la souche commerciale white Leghorn. Sonaiya et Swan (2004) et Hossary et Galal (1995) ont rapporté que les races locales se caractérisaient par des œufs de petit calibre mais dotés d'une proportion élevée en jaune, en comparaison aux souches commerciales.

\section{LE POURCENTAGE DE LA COQUILLE}

Les poules locales avaient présenté le pourcentage de coquille le plus élevé (12,38 \%). Il est significativement $(\mathrm{P}<0,001)$ différent de celui des autres poules. De même Amer (1972) et Merat et al., (1983) et Tixier-Boichard et al., (2006b) ont enregistré des proportions de coquille significativement supérieures à celles des souches commerciales. Cela s'expliquerait par la forte corrélation négative $(-0,44)$ enregistrée entre le pourcentage des coquilles et le poids de l'œuf entier. Jaya Laxmi (2006) a rapporté l'existence d'importantes corrélations génétique $(-0,146)$ et phénotypique $(-0,267)$ entre le pourcentage des coquilles et le poids de l'œuf entier.

\section{LE RAPPORT JAUNE BLANC ET LA FRAICHEUR DE L'OEUF}

\section{LE RAPPORT JAUNE / BLANC}

Les poules locales avaient présenté un rapport Jaune / Blanc le plus élevé $(0,57)$. De même Tixier-Boichard et al., (2006b) et Mérat et al., (1983) avaient rapporté que le rapport Jaune / Blanc de la race locale Fayoumi est supérieur aux souches industrielles. A 55 semaines d'âge, Suk et Park (2001) ont enregistré un rapport Jaune / Blanc de 0,55 chez la race locale KNC (Korean Native Chicken) et 0,38 pour la poule commerciale CEC (Commercial egg-type chicken).

Ces résultats s'expliqueraient par le pourcentage élevé de jaune comparé à celui de blanc chez les races locales comparées aux souches commerciales. Ils peuvent être aussi liés à des gènes responsables de ces disproportions entre les différents composants de l'œuf.

\section{LA FRAÎCHEUR DE L'OEUF}

La fraîcheur de l'œuf est représentée dans cette étude par les unités d'Haugh. Les poules croisées avaient présenté les œufs les plus « frais » $(89,31)$, suivi des poules locales $(86,46)$. Les poules industrielles avaient présenté les œufs les moins frais $(79,65)$.

Les unités d'Haugh varient d'une race à l'autre (Benabdeljelil et al., 2003 ; Hocking et al., 2003 ; Monira et al., 2003 ; Moula et al., 2009 a; Moula et al., 2009b ; Padhi et al., 1998), ce qui expliquerait les différences enregistrées dans cette étude. Offiong et al., (2006), Hocking et al., (2003) et Amer (1972) ont enregistré des "fraîcheurs » supérieures dans les œufs de souches commerciales comparés à ceux des races locales. Cependant, Padhi et al., (1998) ont noté que la souche commerciale étudiée a présenté des œufs plus frais que ceux de trois races locales, mais moins frais qu'une autre race locale, conformément aux résultats de la présente étude. Ces résultats peuvent être expliqués par le fait que la détermination de la fraîcheur par les unités d'Haugh est influencée par la race. En effet Silversides (1994), Scott et Silversides (2000), Silversides et Scott (2001) et Silversides et Budgell (2001) ont rapporté que l'Unité d'Haugh, comme mesure de fraicheur, est biaisée par la race de la poule.

\section{LES COEFFICIENTS de CORRÉlATION de PEARSON}

Des corrélations hautement significatives $(\mathrm{P}<0,001)$ ont été enregistrées entre le poids de l'œuf entier et les poids du blanc $(95,2 \%)$, du jaune $(50,2 \%)$ et de la coquille (68,5\%). En effet, l'augmentation du poids de l'œuf entier s'accompagne d'une augmentation des poids du blanc, du jaune et de la coquille (Mérat et al., 1983). Suk et Park (2001) ont enregistré à 55 semaines d'âge des coefficients de corrélation de 95,2\%, 64,8 \% et $66,8 \%$ entre le poids de l'œuf entier et, respectivement, les poids du blanc, du jaune et de la coquille. Pour leur part, Offiong et al., (2006) ont enregistré une corrélation significative $(\mathrm{P}<0,01)$ de $49,76 \%$ et $55,75 \%$ respectivement entre le poids de l'œuf et le poids du jaune des races exotiques et locales.

Pour les proportions du blanc, du jaune et de la coquille, des corrélations importantes $(\mathrm{P}<0,001)$, respectivement de $79,2 \%,-74,4 \%$ et de $-36,6 \%$, sont enregistrées avec le poids de l'œuf entier. En effet l'augmentation du poids de l'œuf induit une augmentation de la proportion du blanc au détriment des proportions du jaune et de la coquille qui diminuent. Marion et al., (1964) ont observé une corrélation importante et négative entre le pourcentage du jaune et le poids de l'œuf. Cette corrélation négative est liée, selon Moula et al., (2010), à l'augmentation du poids et de la proportion du blanc dans les œufs de grand calibre. Pour leur part, Das et al., (2006) ont noté une corrélation négative du pourcentage de la coquille avec le poids de l'œuf.

Comme le pourcentage du jaune, le rapport jaune / blanc a présenté une forte corrélation négative (-75,3 $\%$ ) avec le poids de l'œuf entier. En effet une très forte corrélation $(\mathrm{P}<0,001)$ de $99,1 \%$ a été notée entre le pourcentage du jaune et le rapport Jaune / Blanc, alors que ce dernier est corrélé négativement (-96,9\%) avec le pourcentage du blanc.

A 55 semaines d'âge, Suk et Park (2001) ont enregistré des coefficients de corrélations entre le rapport jaune / blanc et le poids de l'œuf de -20,3 et de -65,4 $\%$ pour respectivement la souche commerciale et la race locale. Ils ont conclu que les œufs de petit calibre présentaient un rapport jaune / blanc supérieur à celui des œufs de grand calibre.

La force maximale de rupture de la coquille a présenté un coefficient de corrélation significatif avec l'épaisseur de la coquille $(28,0 \%)$ et avec le poids de l'œuf entier (10,1\%).

En conclusion, l'origine génétique des œufs influe de façon significative la composition et la qualité des 
œufs commercialisés. En Algérie, les lignées commerciales largement utilisées ont absorbé le marché des productions avicoles. Cependant, les préférences alimentaires des consommateurs pour les produits issus des animaux locaux peuvent participer à la sauvegarde de la diversité génétique animale locale, comme c'est le cas en Europe.

\section{BIBLIOGRAPHIE}

Agriculture et Agroalimentaire Canada, http://www.agr.gc.ca/poultry/ proe-preg_f.htm, 2006, 'Portrait de l'industrie avicole canadienne', Agriculture et Agroalimentaire Canada.

Amer, M F 1972, 'Egg quality of Rhode Island Red, Fayoumi and Dandarawi'. Poultry Science, vol. 51, pp. 232-38.

Benabdeljelil, K , Lahbabi, S, Bordas, A 2003, 'Animal ResourcesComparison of Crosses Including a Local Breed or an Experimental Line to a Commercial Control for Egg Production in Morocco', Revue d'Elevage et de Medecine Veterinaire des Pays Tropicaux, vol. 56, pp. 193-98.

Benabdelielil, K, Merat, P 1995, 'Comparaison de types génétiques de poules pour une production d'œufs locale: F1 (Fayoumi $x$ leghorn) et croisement terminal ISA au Maroc', Annales de Zootechnie, vol. 44, pp. 313-18.

Benoff, F, Renden, J 1983, 'Divergent selection for mature body weight in dwarf White Leghorns. 2. Maternal determinants of egg size', Poultry Science, vol. 62, pp. 1938-43.

6. - Bessadok, A, Khochilef, I, El Gazzah, M 2003, 'Etat des ressources génétiques de la population locale du poulet en Tunisie', Tropicultura, vol. 21, pp. 167-72.

Das, A K, Sahoo, A K, Misra, S K, Pal, N C, Paul, R K 2006, 'Interdependence of egg quality traits in Rhode Island Red and Australorp Chicken', Journal of Interacademicia, vol. 10, pp. 261-69.

Dottavio, A, Canet, Z, Alvarez, M, Creixell, B, Di Masso, R, Font, M 2001, 'Productive traits in hybrid hens with Fayoumi maternal genotype', Archivos Latinoamericanos de Producción Animal, vol. 9.

Fletcher, D, Britton, W, Pesti, G, Rahn, A, Savage, S 1983, 'The relationship of layer flock age and egg weight on egg component yields and solids content', Poultry Science, vol. 62, pp. 1800-805.

Francesch, A, Estany, J, Alfonso, L, Iglesias, M 1997, 'Genetic parameters for egg number, egg weight, and eggshell color in three Catalan poultry breeds', Poultry Science, vol. 76, pp.1627-31.

Harms, R, Hussein, S 1993, 'Variations in yolk: albumen ratio in hen eggs from commercial flocks', The Journal of Applied Poultry Research, vol. 2, pp. 166-70.

Hartmann, C, Johansson, K, Strandberg, E, Wilhelmson, M2000, 'Onegeneration divergent selection on large and small yolk proportions in a White Leghorn line', Bristish Poultry Science, vol. 41 , pp. 280-86.

Haugh, R 1937, 'The Haugh unit for measuring egg quality'.

Hocking, P, Bain, M, Channing, C, Fleming, R, Wilson, S 2003, 'Genetic variation for egg production, egg quality and bone strength in selected and traditional breeds of laying fowl', Br Poultry Science, vol. 44 , pp. 365-73

Hossary, M, Galal, E 1995, 'Improvementand adaptation of the Fayoumi chicken', Animal Genetic Resources Information, vol. 14, pp. 33-42.

Jaya Laxmy, P 2006, 'Correlations among various egg quality traits in White Leghorn', Indian veterinary journal, vol. 83, pp. 59-62.

Kemps, B, Govaerts, T, De Ketelaere, B, Mertens, K, Bamelis, F, Bain, M, Decuypere, E, De Baerdemaeker, J 2006, 'The influence of line and laying period on the relationship between different eggshell and membrane strength parameters', Poultry Science, vol. 85, pp. 1309-17.

Ketelaere, BD, Govaerts, T, Coucke, P, Dewil, E, Visscher, J, Decuypere, E, Baerdemaeker, J D 2002, 'Measuring the eggshell strength of 6 different genetic strains of laying hens: techniques and comparisons', Br Poultry Science, vol. 43, pp. 238-44.
Marion, W, Nordskog, A, Tolman, H, Forsythe, R 1964, 'Egg composition as influenced by breeding, egg size, age and season', Poultry Science, vol. 43, pp. 255-64.

Merat, P B A, L'Hospitalier, R, Protai, S, Bougon, M 1983, 'Etude des particularit6s de la poule Fayoumi. III. Ponte, caractéristiques des \&oelig; ufs, efficacité alimentaire et paramètres physiologiques de poules Fayoumi, Rhode Island et F, en batteries', Génét. Sel. Evol., vol. 15, pp. 147-66.

Mine, Y 2002, 'Recent advances in egg protein functionality in the food system', World's Poultry Science Journal, vol. 58, pp. 31 1-39.

Mine, Y, Kovacs-Nolan, J 2004, 'Biologically active hen egg components in human health and disease', The Journal of Poultry Science, vol. 41, pp.1-29.

Monira, K, Salahuddin, M, Miah, G 2003, 'Effect of breed and holding period on egg quality characteristics of chicken', Int. J. Poult. Sci., vol. 2, pp. 261-63.

Moula, N, Antoine-Moussiaux, N, Farnir, F, Leroy, P 2009a, 'Comparison of egg composition and conservation ability in two Belgian local breeds and one commercial strain', International Journal of poultry science, vol. 8, pp. 768-74.

Moula N, Antoine-Moussiaux, N, Farnir F, Leroy P 2009b, 'Evaluation of the production performances of an endangered local poultry breed, the famennoise' International Journal of Poultry Science, 8 (4), pp.389-396.

Moula, N, Antoine-Moussiaux, N, Decuypere, E, Farnir, F, Mertens, K, De Baerdemaeker, J, Leroy, P 2010, 'Comparative study of egg quality traits in two Belgian local breeds and two commercial lines of chickens', Arch. Geflügelkunde, vol. 74, pp. 164-71.

Moula, N, Ait Kaki, Leroy, P, Antoine-Moussiaux, N 2013, Quality assessment of marketed eggs in Basse Kabylie (Algeria) Revista Brasileira de Ciência Avícola, vol.15 no.4, pp. 395-400.

Nau, F, Antoin, M, Nys, Y 2003, 'L'œuf de poule: une mine de molécules à activités biologiques', Cinquièmes Journées de la Recherche Aviole, Tours.

Nys, Y, Sauveur, B 2004, 'The nutritional value of eggs', INRA Prod. Anim., vol. 17, pp. 385-93.

Offiong, S, Ojebiyi, O, Moses, E, Umoh, B, Offiong, E 2006, 'Comparison of the morphometric characteristics of exotic commercial and local chicken eggs in the tropical environment', Journal of Animal and Veterinary Advances.

Özbey, O, Esen, F 2007, 'The effects of different breeding systems on egg productivity and egg quality characteristics of rock partridges', Poultry Science, vol. 86, pp. 782-85.

Padhi, M, Rai, R, Senani, S. Saha, S 1998, 'Assessment of egg quality in different breeds of chicken', Indian Journal of Poultry Science, vol. 33, pp. 113-15.

Pandey, N, Mahapatra, C, Verma, S, Goyal, R, Johari, D 1984, 'Physical quality traits and components of egg from different strains of White Leghorn hens', Indian Journal of Poultry Science.

Parmar, S, Thakur, M, Tomar, S, Pillai, P 2006, 'Evaluation of egg quality traits in indigenous Kadaknath breed of poultry', Livestock Research for Rural Development, vol. 18.

Poggenpoel, D, Duckitt, J 1988, ' Genetic basis of the increase in egg weight with pullet age in a White Leghorn flock', Br Poultry Science, vol. 29, pp. 863-67.

Premavalli, K, Viswanathan, K 2004, 'Influence of system of management on the external egg quality characteristics of commercial White Leghorn layers', Indian veterinary journal, vol. 81, pp. 1364-67.

Scott, T, Silversides, F 2000, 'The effect of storage and strain of hen on egg quality', Poultry Science, vol. 79, pp. 1725-29.

Silversides, F, Budgell, K 2004, 'The relationships among measures of egg albumen height, $\mathrm{pH}$, and whipping volume', Poultry Science, vol. 83, pp. 1619-23.

Silversides, F, Scott, T 2001, 'Effect of storage and layer age on quality of eggs from two lines of hens', Poultry Science, vol. 80, pp. 1240-45. 
Silversides, F G 1994, 'The Haugh unit correction for egg weight is not adequate for comparing eggs from chickens of different lines and ages', Journal of Applied Poultry Research., vol. 3, pp. 120-26.

Sonaiya, E, Swan, S 2004, 'Production en Aviculture familiale', Food \& Agriculture Org.

Stadelman, W, Pratt, D 1989, 'Factors influencing composition of the hen's egg', World's Poultry Science Journal (United Kingdom).

Statistical Analysis System (SAS) 1989, 'Sas/stat. User's guide, version 6, 4th edition', Cary, NC: SAS Institute.

Suk, Y, Park, C 2001, 'Effect of breed and age of hens on the yolk to albumen ratio in two different genetic stocks', Poultry Science, vol. 80 , pp. 855-58.

Tharrington, J, Curtis, P, Jones, F, Anderson, K E 1999, 'Comparison of physical quality and composition of eggs from historic strains of single comb White Leghorn chickens', Poultry Science, vol. 78, pp. 591-94.

Tixier-Boichard, M, Audiot, A, Bernigaud, R, Rognon, X, Berthouly, C, Magdelaine, P, Coquerelle, G, Grinand, R, Boulay, M, Ramanantseheno, D 2006a, 'Valorisation des races anciennes de poulets: facteurs sociaux, technico-économiques, génétiques et réglementaire', Les Actes du BRG, vol. 6, pp. 495-520.

Tixier-Boichard, M, Joffrin, C, Gourichon, D, Bordas, A 13-18 August, 2006b, of Conference, 'Improvement of yolk percentage by crossbreeding between a commercial brown-egg layer and a local breed, the Fayoumi', In: Proceedings of the 8th World Congress on Genetics Applied to Livestock Production, Belo Horizonte, Minas Gerais, Brazil, p.^pp, Year, 32-09.

Tyler, G, Geake, F 1964, 'Egg shell strength and its relationship to thickness, with particular reference to individuality in the domestic hen', Br Poultry Science, vol. 5, pp. 3-18.

Washburn, K, Marks, H 1985, 'Changes in egg composition of lines selected for divergence in yolk cholesterol concentration', Poultry Science, vol. 64, pp. 205-11.

Yoo, B, Sheldon, B, Podger, R 1983, 'Genetic parameters for egg weight $v$. age curve, and other egg production and egg weight traits, in synthetic lines of chickens', Crop and Pasture Science, vol. 34 , pp. 85-97. 